\title{
Automated Detection of New Vessels for Classification of Proliferative Diabetic Retinopathy
}

\author{
Israr ul haq ${ }^{\mathrm{a}, \mathrm{b}^{*}}, \mathrm{Usman} \mathrm{Akram}^{\mathrm{c}}$, Yoshifumi Saijo ${ }^{\mathrm{a}}$ \\ ${ }^{a}$ Graduate School of Biomedical Engineering, Biomedical Image Laboratory, Tohoku University, Japan \\ ${ }^{\mathrm{b}}$ Department of Electrical and Computer Engineering, Center for Advanced Studies in Engineering, Pakistan \\ ${ }^{\mathrm{c}}$ Department of Computer Engineering, National University of Sciences \& Technology, Pakistan \\ *Israrul haq: i_raaz@yahoo.com
}

\begin{abstract}
Optic disc is an important anatomical feature of the retinal image and helps in the diagnosis of eye diseases which appears as a bright circular or elliptic shape in fundus image. Accurate detection of the position of optic disc is useful for the diagnosis of new vessels on optic disc. Proliferative diabetic retinopathy is an advance stage of diabetic retinopathy and causes severe visual impairments. Early detection of the diabetic retinopathy is very important for the protection of patient's vision. Automated systems for screening of proliferative diabetic retinopathy should accurately detect the blood vessels to identify vascular abnormalities. In the proposed method the vascular pattern of normal and abnormal vessels is extracted using Gabor wavelet and segmenting the blood vessels using active contour method. Support vector machine is used for the classification of neovascularization. The proposed method is evaluated using publicly available retinal image databases and results show that the proposed method identifies the vascular abnormalities with high accuracy.
\end{abstract}

Keywords: Diabetic retinopathy, Proliferative diabetic retinopathy, Neovascularization, Optic disc.

\section{Introduction}

In healthcare, medical imaging has emerged over the past few years, which has been considered a crucial tool for effective cures and better diagnosis. Medical imaging has enabled physicians to detect diseases at its early stages. Diabetic retinopathy is one of the diseases which appear in diabetic patients in which the lack of insulin causes high blood sugar in humans. Long-term diabetes also affects the human retina resulting in a condition known as diabetic retinopathy. The symptoms started from eye when the vascular system of the eye is damaged, severe vision loss can occur. This condition damages the retinal blood vessels causing them to leak which ultimately leads to blindness. Proliferative diabetic retinopathy is one of the advanced stages where new vessels start growing on the surface of retina. The early detection of these new vessels is important, because it may cause blindness if not detected timely. There are two types of diabetic retinopathy ${ }^{(1,2)}$.

\section{Non-proliferative Diabetic Retinopathy (NPDR) \\ 2. Proliferative Diabetic Retinopathy (PDR)}

1. Non-Proliferative Diabetic Retinopathy: NPDR which is also called background retinopathy (BDR). In BDR the blood vessels in the retina become weakened causing tiny areas of blood protruding from veins or arteries called micro aneurysms ${ }^{(3)}$. These are small blood clots occur due to capillary burst. Due to their small sizes these are hard to detect in retinal images whereas hemorrhages are bigger clots. The micro aneurysms may start leaking fluid into the retina, which may lead to swelling of the macula. Fig. 1 shows the NPDR case.

2. Proliferative Diabetic Retinopathy: PDR is an advanced stage of diabetic retinopathy. At this stage retina sends signal to the brain for new vessels at oxygen deprived areas. These abnormal blood vessels start growing on the surface of the retina and they are fragile and weak which may bleed into the vitreous humor and block the passage of light to the retina resulting in loss of vision or even blindness. Fig. 2 shows the abnormal blood vessels on the optic disc $(\mathrm{NVD})^{(4)}$ or elsewhere $(\mathrm{NVE})^{(5)}$. 


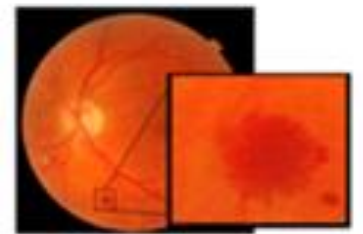

a) Hemorrhage

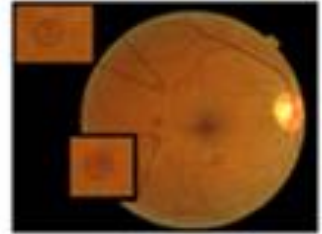

b) Mircoaneursyms
Figure 1. Non-Proliferative diabetic retinopathy
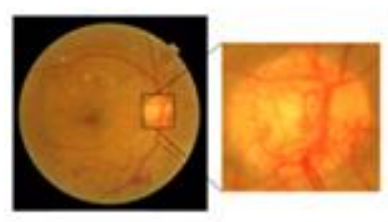

a) NVD

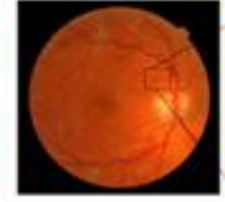

b) NVE
Figure 2. Proliferative diabetic retinopathy

A number of methods have been proposed in literature for the early detection of DR. These methods discussed the complications related to NPDR such as microaneurysms and exudates. The early detection of DR is important to prevent vision loss but the detection of PDR is also very important as it is an advanced stage of DR and only few studies have been carried out on PDR.

Goatman et al. ${ }^{(4)}$ proposed a method to detect new vessels on optic disc by detecting blood vessels. Support vector machine is used to categorize a vessel segment as normal or abnormal. Agruto et al. ${ }^{(6)}$ proposed a multiscale amplitude modulation- frequency modulation (AM-FM) based method for discriminating between normal vessels and neovascularization. Jelinek et al. ${ }^{(7)}$ examined the vascular pattern characteristics to detect PDR on fluorescein angiogram images. Nayak al. ${ }^{(8)}$ proposed an artificial neural network based method for the detection of PDR with the help of blood vessels area and perimeter. The method for the detection of PDR should be able to detect blood vessels accurately and numbers of methods have been proposed for the detection and segmentation of blood vessels but the detection of neovascularization is still a difficult task as these vessels are small and fragile ${ }^{(9-10)}$. Chaudhuri et al. ${ }^{(1)}$ proposed matched filter method for the detection of blood vessels and this method has been widely used for the detection of blood vessels but the method fails to detect small vessels. Threshold probing based method was presented by Hoover et al. ${ }^{(10)}$ to improve the accuracy of matched filter. The methods discussed contain different approaches to detect blood vessels but in this paper a new approach has been used to detect the normal and abnormal blood vessels. Fig. 2 shows the PDR case.

The rest of the paper is organized as follows: Section 2 describes the proposed method and all its phases in detail. The results of the proposed system on retinal images are discussed in Section 3 followed by conclusion in the last section.

\section{Proposed Method}

Uncontrolled diabetic retinopathy and glaucoma may lead to blindness. Optic disc helps to identify the different stages of DR, and glaucoma. The new vessels which are hard to detect in an automated way are detected using Gabor wavelets. The aim of this research is to design and implement an automated system for reliable diagnosis of NVD using state of the art image processing techniques.

Fig. 3 shows the proposed flow diagram.

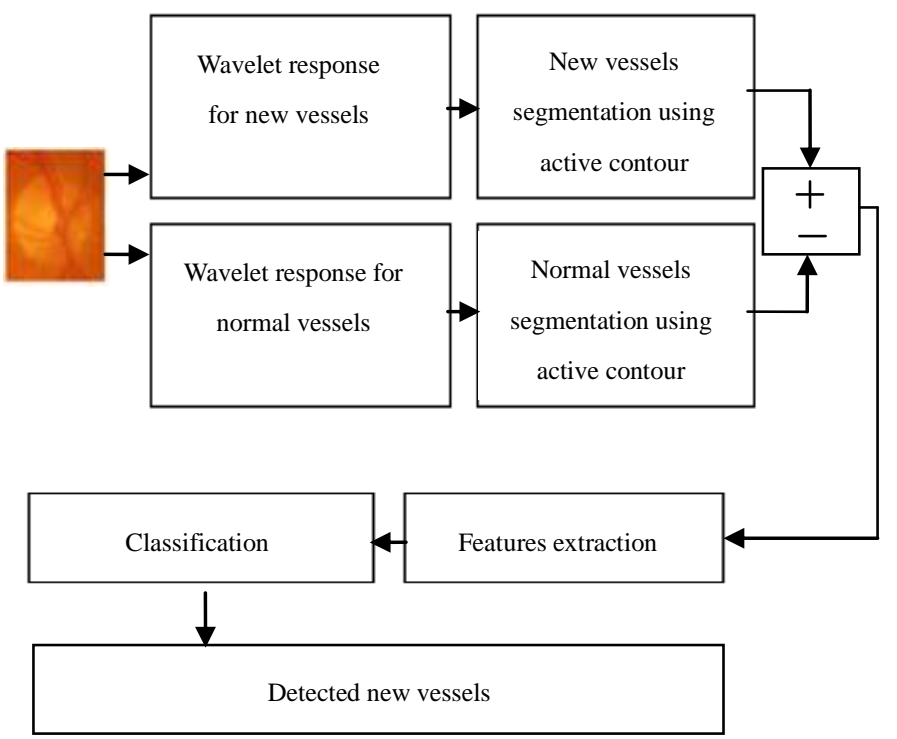

Figure 3. Flow diagram for the detection of NVD

\subsection{Pre-processing and Vessels Segmentation}

Fundus images are acquired using fundus camera. The image quality may be degraded while taking picture. Image quality is an important issue especially in medical imaging. The pre-processing of an image may give false detection if it is not processed correctly. Fundus images contain a circular region of interest on a dark background. Feature extraction and abnormality detection algorithm only need the foreground pixels so a binary mask is used to separate the foreground pixels from the background. The retinal image contains three channels red, green and blue. 


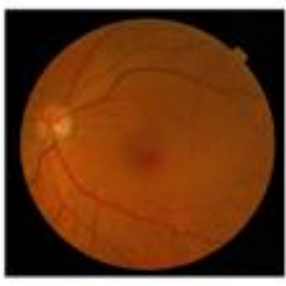

a) Fundus image

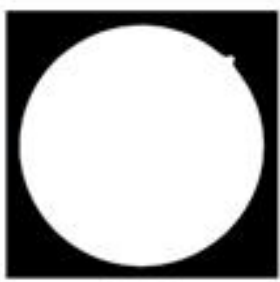

b) Image mask
Figure 4. Retinal image masking

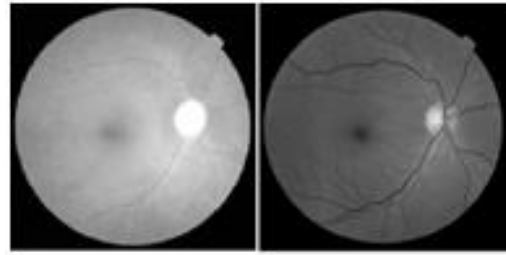

a) Red Channel b) Green Channel

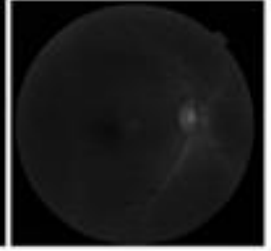

c) Blue Channel
Figure 5. Channels of fundus image

Depending upon the features different channels can be used each contains information of anatomical features e.g. green channel $^{(12)}$ contains information of blood vessels. In this channel vascular structure of vessels can easily be identified and used for processing and red channel contains the information of optic disc. Fig. 5 shows the red, green and blue channels.

\subsection{Vascular Pattern Extraction}

In retinal image analysis Gabor wavelet ${ }^{(13)}$ are considered to be useful for the detection of blood vessels. To enhance the visibility of low intensity vessels 2-D Gabor wavelet is used. Gabor wavelets can be used for thin and thick vessels by selecting the appropriate frequency $\mathrm{k}_{\mathrm{o}}$, dilation parameter $\alpha$, angle $\theta$ and elongation $\epsilon$. Vessels in different directions can be made more visible by adjusting angle and the maximum response is taken at each step. Small step size or angle takes more time to compute the response as compared to large step size. Fig. 6 shows the Gabor wavelet and enhanced blood vessels in retinal image mathematically Gabor wavelet can be expressed as

$$
\psi_{M}(x)=\exp \left(j k_{o} \cdot x\right) \exp \left(-\frac{1}{2}|A x|^{2}\right)
$$

Where $\mathrm{j}=\sqrt{-1}, \mathrm{~A}=\left[\epsilon^{-1 / 2}, 1\right]$ with elongation $\epsilon \geq 1$ is a $2 \times 2$ array and defines the elongation in the particular direction and the frequency of the complex exponential is controlled by vector $\mathrm{K}_{\mathrm{o}}$. Gabor wavelet is scaled by varying the parameters to detect normal and abnormal vessels. Fig. 7 shows the new vessels on optic disc and normal vessels as well. The steps for the detection of new vessels on optic

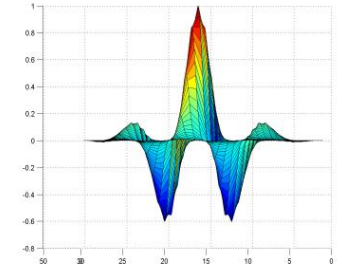

a) Gabor Wavelet

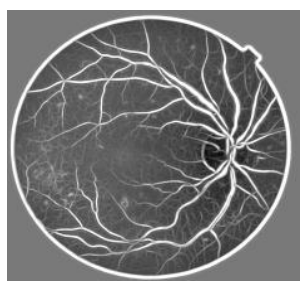

b) Enhanced Image
Figure 6. Effect of gabor wavelet on retinal image
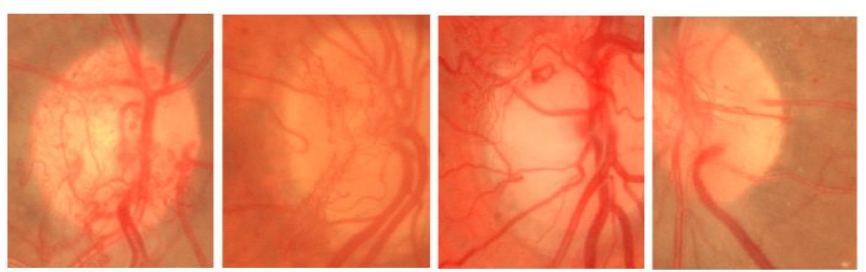

Figure 7: New vessels on optic disc

disc are as follows:

1. The optic disc region is blurred to remove new vessels.

2. Gabor wavelets are applied on the resultant image which gives only normal vessels shown in fig. 8 (b).

3. Active contour method is used to segment the normal vessels

4. From Step: 1 the contrast of optic disc region is enhanced using contrast limited adaptive histogram equalization.

5. Scaled Gabor wavelet is used on the resultant image which gives normal and abnormal vessels shown in fig. 8 (a).

6. Active contour method is used to segment abnormal and normal vessels.

7. Subtract step: 3 from step: 5 it will give abnormal vessels with background noise.

8. Resultant image is passed through the classifier which detects the new vessels and removes the background noise and normal vessels if any.

The optic disc region is blurred using $15 \times 15$ window to remove abnormal vessels and only normal vessels are visible in the resultant image these normal vessels are 
segmented using active contour method or simple thresholding can be used to extract vascular pattern of normal vessels. The same procedure is used on the
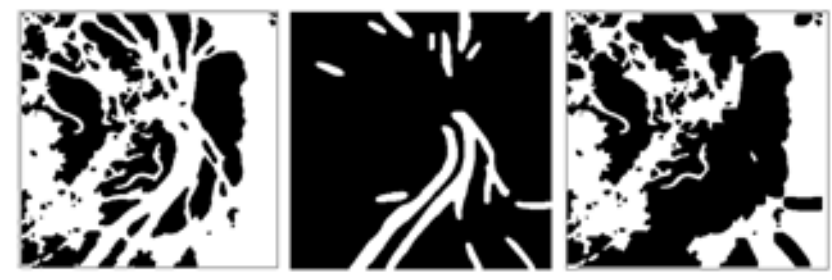

a) New vessels and normal vessels; b) Normal vessels; c) New vessels

Figure 8. Detection of new vessels

same image but with scaled Gabor wavelet so that the wavelet gives response to abnormal vessels. Wavelet is scaled to the size of abnormal vessels and rotations are given at a step size of $5^{\circ}$ so that the bunch of abnormal vessels could easily be extracted. Then the dilated normal vessels are subtracted from abnormal vessels. The resultant image will contain abnormal vessels with background noise. If the normal vessels are not dilated then the boundaries of the normal vessels are also detected as new vessels. Some of the normal vessels may also appear in the subtracted image but these can easily be removed when passed through classifier. Fig. 8 shows the image of new vessels obtained after subtracting.

Detection of abnormal vessels requires the appropriate window size to extract features. If window size is not selected properly than feature values of abnormal vessels may vary because if large window is selected then some portion of the retinal background can also be extracted which may change the value of features and abnormal vessels may not be detected properly. The problem can be solved if different segments of abnormal vessels are labeled in an automated way or selecting a small window size so that only a portion of the abnormal vessel is selected or a bunch of abnormal vessels are selected.

Abnormal new vessels are fragile and have weak walls so the feature selection for classification is itself a big task as some of the vessels appeared in the form of bunch whereas some may appear separate so a window size of $25 \times 25$ is selected in case of Messidor dataset which mainly depends on the resolution of the retinal image. Different window size may give different results, while extracting features appropriate window size should be selected so that features of each small segment could be calculated. Features such as mean gradient, energy, intensity variation and mean intensity are used.

\section{Results}

In order to evaluate the performance of the classifier and output of the overall system four parameters are used for evaluation purpose: i.e. sensitivity, specificity, accuracy and positive prediction value (PPV) given in Eqs. (2) to (5).

$$
\begin{aligned}
\text { Sentivity } & =\frac{T_{p}}{T_{p}+F_{N}} \\
\text { Specificity } & =\frac{T_{N}}{T_{N}+F_{P}} \\
\text { Accuracy } & =\frac{T_{p}+T_{N}}{T_{p}+T_{N}+F_{T}+F_{P}} \\
\text { PPV } & =\frac{T_{p}}{T_{p}+F_{P}}
\end{aligned}
$$

where,

TP (true positive): Pixels that are computed as new vessels and they also belong to new vessels in ground truth.

FP (false positive): Pixels that are computed as new vessels and they are non-vessels in ground truth.

TN (true negative): Pixels that are non-vessels and they are non vessels in ground truth.

FN (false negative): Pixels that are non-vessels and they are computed as new vessels in ground truth.

The performance results for the proposed system are given in table 1.

Table 1. Performance of proposed method for NVD

\begin{tabular}{|c|c|}
\hline Sensitivity (\%) & $83.87_{+/-} 8.08$ \\
\hline Specificity (\%) & $97.62_{+/-} 1.60$ \\
\hline PPV (\%) & $96.48_{+/-} 2.32$ \\
\hline Accuracy $(\%)$ & $92.34_{+/-} 5.34$ \\
\hline
\end{tabular}

The messidor dataset is used for training new-vessels segments. Fig. 9 shows the results of new vessels on optic disc after classification. Fig. 10 shows the results where no abnormal vessels are detected after classification because of healthy retinal images. The green region shows the window size used to crop the area where OD exists.
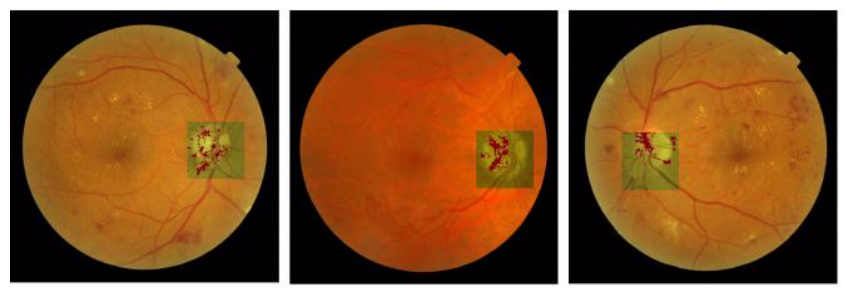

Figure 9: Detected new vessels 

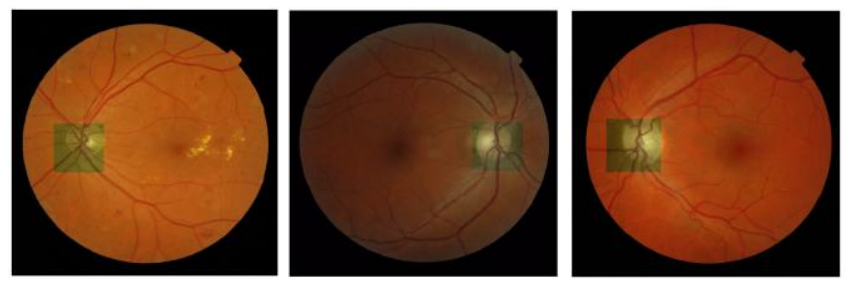

Figure 10: No new vessels detected

\section{Conclusions}

Proliferative diabetic retinopathy is an advance stage of diabetic retinopathy and needs an early detection. In the proposed method novel algorithms for the detection of proliferative diabetic retinopathy is analyzed and the proposed algorithm gave good results with high accuracy on publicly available datasets. In the proposed method active contour method was used to segment the new vessels, it takes number of iterations to converge. Some other method of segmentation and new features to classify the vessels and non-vessels segments can also be used.

\section{References}

1. S. Kanth, A. Jaiswal, and M. Kakkar: "Identification of different stages of Diabetic Retinopathy using artificial neural network", Sixth International Conference on Contemporary Computing, pp. 479 - 484, 2013

2. M. Preethi and R. Vanithamani: "Review of retinal blood vessel detection methods for automated diagnosis of diabetic retinopathy", IEEE-International Conference on Advances In Engineering Science And Management, pp. 262-265, 2012.

3. R. C. Silvia and R. Vijayalakshmi: "Detection of non-proliferative diabetic retinopathy in fundus images of the human retina", International Conference on Information Communication and Embedded Systems, pp. 978-983, 2013.

4. K.A. Goatman, A.D. Fleming, S. Philip, G.J Williams, J. A Olson, and P.F Sharp: "Detection of new vessels on the optic disc using retinal photographs", IEEE Trans Medical Imaging, Vol. 30, No. 4, pp. 972-979, 2011.

5. L. Zhang, Q. Li, J. You, and D. Zhang: "A modified matched filter with double-sided thresholding for screening proliferative diabetic retinopathy", IEEE Transaction on Information Technology in Biomedicine,
Vol. 13, No. 4, pp. 528-534, 2009.

6. C. Agurto, V. Murray, E. Barriga, S. Murillo, M. Pattichis, and H. Davis: "Multi-scale AM-FM methods for diabetic retinopathy lesion detection", IEEE Transaction on Medical Imaging, Vol. 29, No. 2, pp. 502-512, 2010.

7. H. F. Jelinek, M. J. Cree, J. J. G. Leandro, J. V. B. Soares, and A. Luckie: "Automated segmentation of retinal blood vessels and identification of proliferative diabetic retinopathy", J Opt Soc Am A, Vol. 24, No. 5, pp. 1448-1456, 2007.

8. J. Nayak, P. S. Bhat, R. Acharya, U. C. M Lim, and M. Kagathi: "Automated identification of diabetic retinopathy stages using digital fundus images", J Med Syst, Vol. 32, No. 2, pp. 107-115,2008.

9. J. J. Staal, M. D. Abramoff, M Niemeijer, M. A. Viergever, and B. van Ginneken: "Ridge based vessel segmentation in color images of the retina", IEEE Trans action on Medical Imaging, Vol. 23, No. 4, pp. 501-509,2004.

10. A. Hoover, V. Kouznetsova and M. Goldbaum: "Locating blood vessels in retinal imagesby piecewise threshold probing of a matched filter response", IEEE Transcation on Medical Imaging, Vol. 19, No. 3, pp. 203-210, 2000.

11. S. Chaudhuri, S. Chatterjee, N. Katz, M. Nelson, and M. Goldbaum: "Detection of blood vessels in retinal images using two-dimensional matched filters", IEEE Transcation on Medical Imaging, Vol. 8, No. 3, pp. 263-269,1989.

12. H. Yu, E. S. Barriga, C. Agurto, S. Echegaray, M. S. Pattichis,W. Bauman,,and P. Soliz: "Fast localization and segmentation of optic disk in retinal images using directional matched filtering and level sets", IEEE Transactions on Information Technology in Biomedicine, Vol. 16, No. 4, pp. 644-657, 2012.

13. M. U. Akram, A. Atzaz, S. F. Aneeque, and S. A. Khan: "An unsupervised segmentation method for retinal vessel using combined filters", International Conference on Digital Image Processing, Vol. 1, pp. 34-38, 2009. 